\title{
A Geometry Consisting of Singularities Containing Only Integers
}

\author{
Qing $\operatorname{Li}^{1}$ \\ ${ }^{1}$ ShiJiaZhuang Traditional Chinese Medical Hospital, China \\ Correspondence: Qing Li, ShiJiaZhuang Traditional Chinese Medical Hospital, ShiJiaZhuang, HeBei province, \\ China.
}

Received: January 7, 2022

Accepted: February 18, 2022

Online Published: February 23, 2022

doi:10.5539/cis.v15n2p38

URL: https://doi.org/10.5539/cis.v15n2p38

\begin{abstract}
It is difficult for us to discriminate the sizes of space and time as finite and infinite. In this article, an axiom is defined in which one infinitely small and infinitely great must exist if the sizes of space and time can be compared and it is undividedly 0 (zero) point (singularity) for this infinitely small. This axiom has some new characters distinct from current calculus, such as extension only can be executed in the way of unit superposition in the system, the decimal point is meaningless and there are only integers to exist in the system, and any given interval is finite quantities and cannot be 'included' or 'equal divided' infinitely and randomly. The geometry space we see is the non-continuum being made of countless 0 points.
\end{abstract}

Keywords: singularity, infinitesimal, units' superposition, decimal point, integer

\section{MSC code: 51-XX and 11-XX}

\section{Introduction}

Infinitesimally small in calculus is defined as infinite quantities close to zero, and we still do not know if such zero points indeed exist (Pysialk L., Sasin W., \& Heller M. 2020) (Hughes D. 2010) . If it exists, what is its relationship to the quantities around it. Moreover, it is difficult for us to define which part is infinitesimal and how to differentiate the sizes of space and the time as finite and infinite. For example, compared with infinitely great, one meter long or 300,000 kilometers is usually regarded as the quantities of finite. However, it can be supposed to be the quantities of infinite due to it is divided infinitely into smaller parts. Thus, it can be seen that we are obscure in the concept of infinitely small and infinitely great. The major contribution of this research concentrates on establishing a concept of axiom 1 where the decimal point is meaningless and there are only integers to exist in the system, and extension of quantities only can be executed in the way of unit superposition in the system. The remainder of the paper is as follows. Section 2 gives the basic concept of axiom 1. Section 3 discusses some basic characteristics of axiom 1. Section 4 presents the future applications of axiom 1 in describing spatial features. Section 5 makes a summary of this paper.

\section{Preliminaries}

\subsection{Definition}

Here an axiom is defined (axiom1) in which one infinitely small and infinitely great must exist if the sizes of space and time can be compared (namely there is small and great in sizes) and it is undividedly 0(zero) point (singularity) for this infinitely small. Now considering this infinitely small.Because it is the minimum quantity, the quantity next to this minimum quantity, that is, the second quantity, is the second 0 , and so on, the third 0 , the fourth 0 , , etc. Therefore, all quantities are in the units of 0 , in order to describe briefly, ' 0 ' is expressed by ' $\mid$ ', as shown in Figure 1. The process of the extension from infinitely small to infinitely great is indicated in this figure.

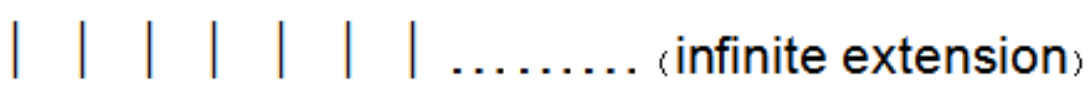

Figure 1. In order to describe briefly, '0'is expressed by' $\mid$ '. The process of the extension from infinitely small to infinitely great is indicated in this figure. 


\subsection{Definition}

Now we define the axiom 2 that is a counter-example toward axiom 2 . Any given system in axiom 2 can be divided infinitely and randomly. The quantities inside this given system (e.g., 1 meter long or 300,000 kliometers) contain all quantities outside the given system and random divided value outside the given system can be found wholly within the system. Contrary to axiom 1 , there is no infinitely small and infinitely great in the axiom 2 . There is no existence of the third axiom. In the event of axiom 1 exists, then axiom 2 do not exist.

\section{Main Results}

\subsection{Preliminaries}

Before some characters of axiom1 are described, some algorithms are adopted to illustrate conveniently the relation among these quantities. The relation between two quantities is described by the terms called 'include' and 'equal divided'.

\subsection{Example}

For example, considering 10 and 2, how much 10 includes 2 is expressed as 10 ( ) $2=5$, and how much 10 is equally divided by 2 is expressed as $10 / 2=5.10$ ( ) $2=5$ is not the same as $10 / 2=5$, because the former can be expressed as $10 / 5=2$ by 'equal divided' and the latter can be expressed as 10 ( ) $5=2$ by 'include'.

\subsection{Theorem}

We find that the extension only can be executed in the way of unit superposition in the system of axiom 1 .

\subsection{Example Now}

we consider two random quantities extending (or superposing) based on the same proportion in the system of axiom1. For example, the accurate value for any extension of 2 ( ) 1 cannot be found due to its next extension 3

() ? have no quantities to be selected within the range of the values in the system, because the infinitely small is smallest and undivided so that there is not value between 1 and 2. For another instance, the accurate value for 4 ( ) 3 cannot be found due to this value only can be selected in 1 and 2 within the range of the values in the system, so the extension of 4 ( ) 3 is meaningless. Therefore, further that is in a unit of $1,2 、 3 、 4 \cdots \cdots$, corresponding to the 'include' values are 1 () $1 、 2$ () 1,3 () 1,4 () $1 \cdots \cdots$. For example, the next extension quantities for 2() 1 is 4() 2 , again the next is 6() 3, and so on. For another instance, the next extension quantities for 5 () 1 is 10 ( ) 2, again the next is 15 ( ) 3, and so on. The 'include' quantities of the extension of the origin must be any units ( ) 1 due to the extension only can be executed in the way of unit quantities, as shown in Figure 2.

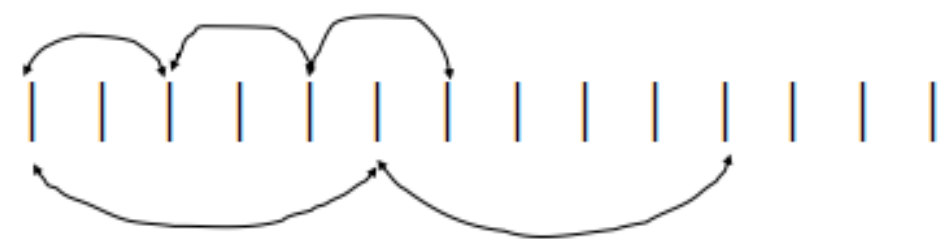

Figure 2. The extension only can be executed in the way of unit superposition in the system. the next extension quantities for 2( ) 1 is 4( ) 2, again the next is 6( ) 3, and so on. .For another instance, the next extension quantities for 5 ( ) 1 is 10 ( ) 2,again the next is 15 ( ) 3, and so on

\subsection{Theorem}

We conclude that there are no definite values for two random quantities that cannot be exactly divided. We let divisor not move (no extension) and allow dividend extend continually to acquire quantities that can exactly divide divisor (Wrobe M. 2020)). It is concluded here that for any two quantities (dividend and divisor) that are not divided exactly we must obtain the aliquot quantities that is integral times of this divisor by the way of extending dividend to infinitely great, not to infinitesimal.

\subsection{Example}

In Figure 3, we consider 4 ( ) 3=? in the range of system of axiom 1. No quantities can be chosen for the accurate value (between 1 and 2) calculated due to 1 and 2 can be only selected in the system. To calculate the accurate value for 4 ( ) 3,3 is not allowed to extend (motionless), and 4 begins extending, following 7, 8, 40, 400, 4000, $4000 \cdots \cdots$. It is random for this extension and thus looking for the accurate value for 4 ( ) 3 in this system has lost meaning. However, the accurate value for $4000000 \cdots \cdots$. ( ) 3 can be found in this system and this value is in the 
unit of 3 , which is quantities that can be divided exactly by 3 .

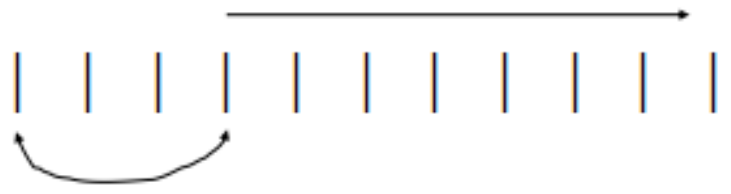

Figure 3. To calculate the accurate value for 4 ( ) 3,3 is not allowed to extend (motionless), and 4 begins extend. The accurate value for $4000000 \ldots \ldots$. ( ) 3 can be found in this system and this value is in the unit of 3

\subsection{Theorem}

It is known from character 2 that the decimal point is meaningless in the system because the decimal point indicates the quantities that cannot be exactly divided. This means that only integer exists, and fraction and irrational number do not exist in the system. Therefore, the basic characteristics of space geometry and the basic characteristics of number theory are equivalent in essence (Burungale A.A., Kobayashi S, \& Ota K. 2021) (Parry W., \&Mark. M. 1983), where number theory and geometry can form a unity (Dospinescu. G., \&Bras, A.C.L. 2017)) (Bushnell C.J., \& Henniart.G. 2017).

\subsection{Example}

Taking an example, for Fermat's big theorem $x^{n}+y^{n}=z^{n}$, since there are no decimal points to exist, its non-integer solutions are meaningless (Wiles A. 1995), so integer solutions must exist. We will give the exact integer solutions of the Fermat's theorem in the next paper.

\subsection{Theorem}

We draw the conclusion from character 2 that it is meaningless to compare with two quantities that dividend is less than divisor.

\subsection{Theorem}

Seeing Figure1. The existence of the infinitely small determines that the number superposition is in the unit of superposition and this means that superposition is truncated one. Thus, we draw the conclusion that the system of axiom 1 is non-continuum.

\subsection{Theorem}

A given interval in the system cannot be 'included' or 'equal divided' infinitely and randomly. Moreover, it is non-existent that the whole quantities can be contained within any given system and for any two given dividend and divisor, which is not divided exactly. Therefore, we must obtain the aliquot quantities that is integral times of this divisor by the way of extending dividend to infinitely great, not to infinitesimal.

\subsection{Example}

For example, in the extent of 20, 20 can only be 'included' or 'equal divided' by 1,2,5,10. This mean that a given interval is finite quantities, for instance, 1 meter long or 300,000 kilometers should be regarded as the finite quantities. Taking another example, still considering 4() $3=$ ?, the accurate value for 4 ( ) 3 cannot be found in the system of axiom 1, but the accurate value for $4000000 \cdots \cdots$. ( ) 3 can be found in this system and this value is in the unit of 3 . Assuming this value is $13333 \cdots \cdots$, then at this moment there are $4000000 \cdots \cdots \cdot 13333 \cdots \cdots=3$, within the range of $4000000 \cdots \cdots$, one quantity $13333 \cdots \cdots 4$ that is beside $13333 \cdots \cdots$ cannot be divided exactly by $4000000 \cdots \cdots$. Therefore, the defined value for $4000000 \cdots \cdots / 13333 \cdots \cdots 4$ cannot be found in the range of $4000000 \cdots \cdots$, you must extend continuously from $4000000 \cdots \cdots$ to more amount quantities to obtain the defined value for the integral times of $13333 \cdots \cdots 4$. At the same time, the defined value of the circular constant $\pi$ cannot be found within any given circumferential length, it can only be found in the quantities more than this given circumferential length by the way of extending this given circumferential length to infinitely great. As a result, this rule can be executed for random ratio quantities in the system of axiom 1.

\subsection{Theorem}

It is inferred from the uniqueness of infinitely small and infinitely great and character 6 that the formula for 1 ( ) $0=\infty$ ( ) $1=\infty$ is non-existent and only $\infty$ ( ) $0=\infty$ is existent in axiom1. Here ' $\infty$ ' indicates infinitely great.

\subsection{Corollary}

It is the sample of axom2 in which some length of space or time, such as 1 meter long, can be divided infinitely 
and randomly in common sense. Meanwhile, it is endowed in this sample by us that the sizes of space and time can be compared (namely there are small and great sizes) in which axiom 1 and axiom 2 are co-existence(mixed). Some characters can be seen in this mixed axiom 1 and axiom 2 , such as the definite values of randomly 'include' and 'equal divided' inside and outside any given system can be found wholly inside this system, the decimal point has meaning, and two random quantities can be extended (enlarge and reduce) randomly based on the same proportion.

\subsection{Example}

For instance, $1.3333 \cdots \cdots$ and $1.3333 \cdots \cdots 4$ are meaningful and can be found within one system that can be applied for any decimal and integral quantities within it. Taking an example, any decimal quantities can be included within any given system, such as the range of one meter long or 300,000 kilometers.

\subsection{Corollary}

Therefore, we conclude that differential and integral calculus, which is a continuous calculation of dividend and divisor based on this mixed axiom 1 and axiom 2, have become not suitable because of the existence of axiom 1 ( Sellés M. A. 2006) ) ( Maderna E., \&Venturelli. A. 2020).

\subsection{Theorem}

From III1-16, some formula can be derived.

$$
\begin{array}{cll}
\lim _{z \rightarrow \infty} \frac{Z}{\mathrm{~m}}=I \quad \mathrm{Z} \mid \mathrm{m}=\mathrm{a} \quad \mathrm{I} \neq \mathrm{a}(\text { non-a) } & (\mathrm{Z}>\mathrm{m}) \\
\lim _{z_{1}, z_{2} \rightarrow \infty} \frac{Z_{2}}{Z_{1}}=I & Z_{2} \mid Z_{1}=\mathrm{b} \quad \mathrm{I} \neq \mathrm{b}(\text { non-b) } & \left(\mathrm{Z}_{2}>\mathrm{Z}_{1}\right) \\
\frac{\mathrm{m}_{2}}{\mathrm{~m}_{1}}=I \quad \mathrm{~m}_{2} \mid \mathrm{m}_{1}=\mathrm{c} \quad \mathrm{I} \neq \mathrm{c}(\text { non-c) } & \left(\mathrm{m}_{2}>\mathrm{m}_{1}\right)
\end{array}
$$

Here $\mathrm{Z}$ is a positive integer (the number of 0 ) and $\mathrm{m}$ is a positive integer with a finite value, noticing that $\mathrm{Z}$ is greater than $m, Z_{2}$ is greater than $Z_{1}$, and $m_{2}$ is greater than $m_{1 .,}$ which are based on Definition II.1. $Z \mid m$ indicates that $Z$ is divided exactly by $m . Z_{2} \mid Z_{1}$ indicates that $Z_{2}$ is divided exactly by $Z_{1} \quad m_{2} \mid m_{1}$ indicates that $m_{2}$ is divided exactly by $\mathrm{m}_{1} . \mathrm{I} \neq \mathrm{a}, \mathrm{I} \neq \mathrm{b}, \mathrm{I} \neq \mathrm{c}$ indicates that they are decimals and have no meaning in axiom 1 .

In Formula $1, Z \rightarrow \infty$ can be regarded as a point at infinity $\mathcal{O}$. When we regard positive integers from 1 to $Z_{1}$ as a set group, we define this group as $\mathbb{Z}_{1}$, here $a_{1}=Z_{1} / m$, and $a_{1} \in \mathbb{Z}_{1}$. Similarly, when we regard positive integers from 1 to $Z_{2}$ as a set group, we define this group as $\mathbb{Z}_{2}$, here $\mathrm{a}_{2}=Z_{2} / \mathrm{m}$, and $\mathrm{a}_{2} \in \mathbb{Z}_{2}$. It is not difficult to see that $\mathrm{a}_{1}$ is not equal to $\mathrm{a}_{2}\left(\mathrm{a}_{1} \neq \mathrm{a}_{2}\right)$, and $\mathbb{Z}_{1} \neq \mathbb{Z}_{2}$, that is, they belong to different $\mathcal{O}$ points. Therefore, from this point of view, whether $Z_{2}$ in formula 2 can be divided exactly by $Z_{1}$ can be determined from axiom 1 .

\section{Discussion}

Consequently, the space we see is made of countless 0 points of non-continuum despite dimensions of space. There are the third implications here:

Firstly, in the definition of axiom 2, a given system, such as 1 meter long, can be divided into infinite smaller quantity, which means that the division can continue forever and do not stop, namely, there is no minimum to exist. However, in axiom 1, because of the existence of the minimum quantity (o point), 1 meter is considered as a finite quantity and cannot be divided infinitely.

Secondly, the existence of decimal points is based on the concept of axiom 2 where the all quantities (including decimals) can be included in any given system (e.g., 1 meter long), so the number of decimal places and their numerical values here are independent of whether they are in or out of a system(Guillarmou C., \&Lefeuvre T. 2019). However, in axiom 1, the decimal point is meaningless and there is only integer, which indicates any new quantity (i.e., algebra and geometry) distribution feature. For example, Considering the integral times of 0 in axiom 1 , such as $21,212,2124,21247 \cdots \cdots$, cannot be included and distributed within one system. They belong to different systems, so in axiom 1, the size of the quantities determines the size of the system to which it belongs.

Thirdly, these two aspects above are not limited to one dimension and zero dimension.

To acquire all definite values in different sizes of space and time is the fundamental goal for mathematics. Some 
readers might ask if the existence of axiom 1 has some help to this goal? For example, 1 meter long, is speculated not to be infinitely great in a common sense because of the existence of the quantities that is large than it. It is known from axiom 1 that 1 meter long is a finite quantity, and then how much is its definite value within the system? For another instance how much 4 ( ) 3 and circular constant $\pi$ ? Some readers ask as well, infinitely small in axiom 1 is 0 (zero) point, that is point of 0 (zero) dimension, and then in what position in the system of axiom 1 is a straight line, plane surface, and even multidimensional curved surface we can see in common sense? The whole questions relate to the illustration of the infinitely great in axiom 1 .

Someone will doubt the validity of axiom 1 , because space and time are continuums, which we see in common sense, is not non-continuum. In addition, it is obscure for us to understand the process of the extension from infinitely small to infinitely great. Therefore, in the next paper, we will answer the above posed questions and try to give the definite values of each length or scale of space and time, particularly concerning the define values of infinitely great.

\section{Conclusions}

In this paper, an axiom is defined in which one infinitely small is undividedly 0 (zero) point (singularity). This axiom 1 has some new characters distinct from current calculus, mainly on the concept that decimal point is meaningless. Moreover, any given interval is finite quantities and cannot be 'included' or 'equal divided' infinitely and randomly. We conclude that the geometry space is the non-continuum being made of countless such 0 points.

\section{References}

Burungale, A. A., Kobayashi, S., \& Ota, K. (2021). Rubin's conjecture on local units in the anticyclotomic tower at inert primes. Ann Math, 194(3), 943-966. https://doi.org/10.4007/annals.2021.194.3.8

Bushnell, C. J., \& Henniart, G. (2017). Higher ramification and the local Langlands correspondence. Ann Math, 185(3), 919-955. https://doi.org/10.4007/annals.2017.185.3.5

Dospinescu, G., \&Bras, A. C. L. (2017). Revêtements du demi-plan de Drinfeld et correspondance de Langlands. Ann Math, 186(2), 321-411. https://doi.org/10.4007/annals.2017.186.2.1

Guillarmou, C., \& Lefeuvre, T. (2019). The marked length spectrum of Anosov manifolds. Ann Math, 190(1), 321-344.

Hughes D. (2010). A minimal classical sequent calculus free of structural rules. Ann Pure Appl Logic, 161(10), 1244-1253. https://doi.org/10.1016/j.apal.2010.03.001

Maderna, E., \&Venturelli, A. (2020). Viscosity solutions and hyperbolic motions: a new PDE method for the N-body problem. Ann Math, 192(2), 499-550. https://doi.org/10.4007/annals.2020.192.2.5

Parry, W., \& Mark, M. (1983). An analogue of the prime number theorem for closed orbits of Axiom A flows. Ann Math, 118(3), 573-591. https://doi.org/10.2307/2006982

Pysialk, L., Sasin, W., \& Heller, M. (2020). Functorial Differential Spaces and the Infinitesimal Structure of Space-Time. Rep Math Phys, 85(3), 443-454. https://doi.org/10.1016/S0034-4877(20)30046-X

Sellés, M. A. (2006). Infinitesimals in the foundations of Newton's mechanics. Hist Math, 33(2), 210-223. https://doi.org/10.1016/j.hm.2005.04.002

Wiles, A. (1995). Modular elliptic curves and Fermat's Last Theorem. Ann Math, 141(3), 443-552. https://doi.org/10.2307/2118559

Wrobe, M. (2020) Divisor class groups of rational trinomial varieties. J Algebraic Geom, 542, 43-64. https://doi.org/10.1016/j.jalgebra.2019.09.021

\section{The data availability statement:}

The [DATA TYPE] data used to support the findings of this study are included within the article.

\section{Copyrights}

Copyright for this article is retained by the author(s), with first publication rights granted to the journal.

This is an open-access article distributed under the terms and conditions of the Creative Commons Attribution license (http://creativecommons.org/licenses/by/4.0/). 